\title{
Body-Mass-Index beeinflusst die Interferon-Wirkung
}

Fragestellung: Hat der Body-Mass-Index (BMI) Einfluss auf die klinische Wirksamkeit der Interferone bei der Behandlung von Patienten mit Multipler Sklerose (MS)?

Hintergrund: Alle Immuntherapien, die für die Behandlung der MS zugelassen sind, werden in einer identischen Dosierung gegeben. Eine gewichts- oder BMI-adaptierte Dosierungsanpassung wird eigentlich - von Immunglobulinen abgesehen - nie vorgenommen. Das hat weniger mit der fehlenden Notwendigkeit zu tun als vielmehr mit der Tatsache, dass für die Zulassung von Immuntherapien in der MS von den Zulassungsbehörden bisher zwar mindestens zwei Phase-III-Studien gefordert werden, aber keine Dosis-Wirkungs-Studien, die sehr aufwendig und teuer wären. Das Ergebnis dieser „Entwicklungspolitik“ bei Immuntherapeutika ist aber, dass alle Patienten, unabhängig von Gewicht, BMI oder anderen metabolischen Faktoren mit identischen Dosierungen be-

Kvistad SS, Myhr KM, Holmøy T et al. Body mass index influence interferon-beta treatment response in multiple sclerosis. J Neuroimmunol 2015; 288: $92-7$ handelt werden. Dass dabei ganz unterschiedliche Effekte zu Tage treten können, liegt eigentlich auf der Hand. Mit anderen Worten: im günstigsten Fall sind Patienten mit einem geringen Körper- gewicht beziehungsweise geringem BMI überdosiert und Patienten mit einem hohen Gewicht unterdosiert.

Patienten und Methodik: In dieser Studie untersuchten Kollegen aus Norwegen den Einfluss des BMI auf die Wirksamkeit von Interferon beta 1a. Dazu untersuchten sie $86 \mathrm{MS}$-Patienten, die Interferon beta 1a über einen definierten Zeitraum erhielten, und unterteilen die Kohorte in zwei Gruppen: Patienten mit einem BMI unter 25 und Patienten mit einem BMI über 25. Vergleichsparameter waren die MRT-Aktivität sowie der Prozentsatz der Patienten ohne jegliche Erkrankungsaktivität (NEDA).

Ergebnisse: In der Gruppe der übergewichtigen Patienten mit einem BMI > 25 zeigten $80 \%$ der Patienten eine MRT-Aktivität, gegenüber nur $48 \%$ der Patienten in der normal- beziehungsweise untergewichtigen Gruppe. Das klinische Kriterium „no evidence of disease activity“ (NEDA) erfüllten ferner nur $13 \%$ der Patienten in der Gruppe der übergewichtigen Patienten, aber immerhin $26 \%$ der Patienten in der Gruppe der Normal- beziehungsweise untergewichtigen Patienten.

Schlussfolgerung: Die norwegischen Kollegen schlossen hieraus, dass der BMI die Wirksamkeit des Interferons maßgeblich beeinflusst.

\section{- Kommentar von Volker Limmroth, Köln}

\section{Pharmakologie 6. Semester}

Ein Pharmakologe würde wahrscheinlich sagen: Ja und? Eigentlich ist es kaum zu fassen, dass wir immer noch alle MSPatienten mit den gleichen Dosierungen behandeln, egal ob sie $45 \mathrm{~kg}$ oder $145 \mathrm{~kg}$ wiegen. Selbst die unterschiedlichen Dosierungen von Rebif 22/44 waren initial nicht dazu gedacht, zumindest zwei gewichtsadaptierte Dosierungen zur Verfügung zu haben, sondern sollten (wie wir alle wissen) nach Erkrankungsaktivität ausgewählt werden. Pharmakologisch ist das eigentlich unhaltbar und kaum zu glauben, dass diese Studie erst 20

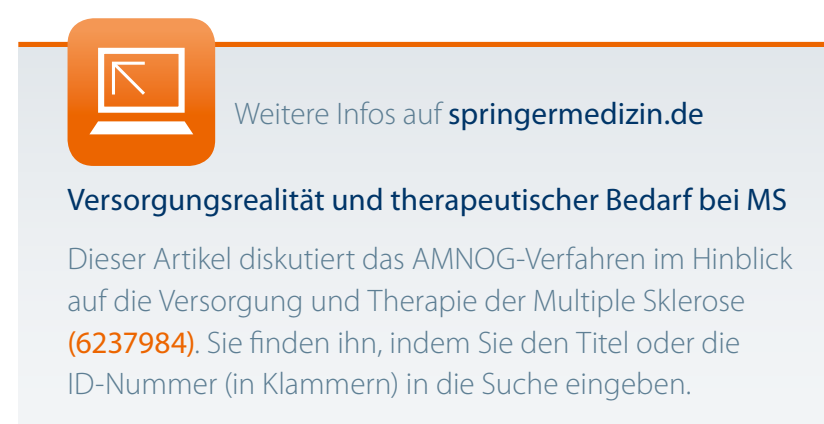

Jahre nach der Zulassung des ersten Interferonpräparates kommt. Es ist zwar irgendwo verständlich, dass die Auflagen für Phase-III-Studien nicht noch komplizierter werden sollten, aber zum Wohle der Patienten, zur Optimierung des Therapieerfolgs und zur Reduktion von Nebenwirkungen wären diese DosisWirkungs-Studien eigentlich dringend erforderlich. Mehr als 20 Jahre nach der Zulassung der Interferone in der Therapie der MS wissen wir nun, was wir immer geahnt haben: Die Wirkung ist auch hier gewichtsabhängig, wie bei allen anderen Medikamenten auch - Pharmakologie 6. Semester.

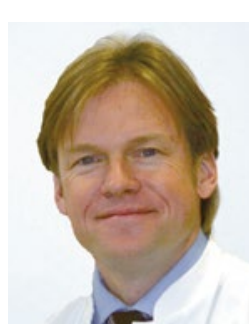

Prof. Dr. med. Volker Limmroth, Köln-Merheim

Chefarzt der Klinik für Neurologie und
Palliativmedizin Köln-Merheim
E-Mail: LimmrothV@kliniken-koeln.de 